\title{
L'utilisation des SIG dans la modélisation en hydrologie de surface
}

\begin{abstract}
From a methodological point of view, hydrological modelling appears to offer a great potential for spatial analysis. However among classes of models, only physical models consider the spatial dimension as a variable. The topography and the land cover are the two major spatial components involved in that type of models. The production of a relevant DTM requires the use of iterative interpolation procedures. Land cover and its changes can be monitored and entered into modelling from remote sensing images through a normalized vegetation index. The GIS approach can be integrated at different levels within hydrological modelling, with object oriented GIS seen as the highest level.
\end{abstract}

\section{Key words}

GIS, hydrological models, DTM, normalized vegetation index, object oriented modelling.

\section{Introduction}

La gestion des ressources en eau, la conception et la réalisation d'aménagements du territoire qui la concernent font largement appel aux connaissances et aux méthodes de l'hydrologie. En effet, les événements climatiques de ces dernières années ont mis en évidence, dans de nombreux endroits de la Suisse ou d'autres pays, la vulnérabilité de certains tronçons de cours d'eau ou d'ouvrages construits pour aménager leur lit ou leurs berges. D'autre part, la qualité des eaux de nos lacs et rivières doit être surveillée avec rigueur pour éviter toute catastrophe écologique. L'hydrologie s'est attachée, d'une part, à comprendre l'ensemble des phénomènes qui lient les précipitations survenant sur un bassin versant et les débits mesurés à son exutoire, d'autre part à la mise au point de modèles de simulation du comportement des bassins versants afin d'établir des prévisions sur les effets probables d'événements climatiques exceptionnels ou non.

Dans quelle mesure l'analyse spatiale à l'aide de systèmes d'information géographique (SIG) contribue à une utilisation plus souple, plus efficace des modèles de simulation ou mieux, à la conception éventuelle de nouveaux modèles plus performants? La tentative de réponse à cette question nécessite la connaissance des méthodes d'approche de l'hydrologie, la mise en évidence des composantes de l'information faisant appel à la dimension spatiale et finalement une réflexion sur l'apport de l'inté- gration du SIG dans la démarche de l'hydrologue, ainsi que sur les finalités de l'analyse.

\section{Les modèles hydrologiques}

Il y a de nombreuses manières de caractériser les modèles hydrologiques. Pour notre propos, nous retenons deux critères: la discrétisation spatiale et la part qu'occupent les paramètres à signification physique dans le modèle.

Ces deux points de vue donnent naissance à des couples opposés de modèles:

- les modèles globaux opposés aux modèles régionalisés

- les modèles conceptuels opposés aux modèles «boite noire»

Les modèles dits à «boîte noire» ne font intervenir aucune information sur la physique du bassin. Ils se contentent d'établir une relation fonctionnelle entre l'événement pluvieux et le débit mesuré à l'exutoire. Les algorithmes s'inspirent de la théorie des signaux: la pluie est un signal d'entrée transformé par le bassin versant en signal de sortie, en débit à l'exutoire. Leur mise en œuvre requiert des séries chronologiques, c'est-à-dire un catalogue représentatifd'événement pluie-débits permettant l'extraction de la fonction de transfert. Ils ignorent de ce fait la dimension spatiale.

Au contraire, les modèles conceptuels (ou à base physique) tâchent de reproduire les mécanismes physiques du système étudié et s'expriment, dans la mesure du possible par des équations de l'hydraulique. Le sens physique donné aux paramètres est un avantage décisif lorsqu'il s'agit de comprendre les changements de comportement d'un bassin lorsque les modifications y sont apportées telles que l'imperméabilisation du sol ou les corrections de cours d'eau.

Les modèles globaux reposent sur l'hypothèse simplificatrice «que le bassin réagit dans son ensemble» à l'événement climatologique (ROCHE 1986).

Bambang Abednego, Dr, Régis Caloz, Dr: Institut d'Aménagement des terres et des eaux, Hydrologie et Aménagements, EPF-L, 1015 Lausanne.

Claude Collet, Dr: Institut de Géographie, Université de Fribourg, Faculté des Sciences, 1700 Fribourg. 
Les paramètres introduits caractérisent l'ensemble du bassin sans tenir compte de la répartition spatiale et de la position respective des différentes zones constituant le bassin versant. On classe dans cette catégorie les modèles à réservoirs en cascade ou celui de l'hydrogramme unitaire. La mise en œuvre de ce type de modèles recourt à des séries chronologiques. On suppose que le bassin n'a pas subi de transformation si importantes (par exemple, une extension très notable des surfaces imperméables) que son comportement probable s'en trouverait fortement influencé. De ce fait, ils ne sont guère utiles pour simuler les effets éventuels d'aménagements projetés sur le bassin versant (remaniements parcellaires, urbanisation, etc...)

Les modèles régionalisés, au contraire, prennent en compte la répartition spatiale des classes d'occupation du sol. Dans son principe, le modèle refléterait les écoulements de chaque zone et les transferts de l'une à l'autre quelle que soit leur forme géométrique. A l'heure actuelle, on ne connaît guère de modèles répondant rigoureusement à ce concept du fait, probablement de la difficulté évidente de maitriser les aspects géométriques. Pour la contourner, on a proposé des modèles conceptuels de type maillés. La discrétisation est alors régulière. Le bassin est divisé en mailles à la manière du monde grille bien connu en SIG. Chaque maille est décrite par des paramètres physiques. Les écoulements sont calculés au moyen d'équations de l'hydraulique à travers chaque maille et d'une maille à l'autre (Fig. 1).

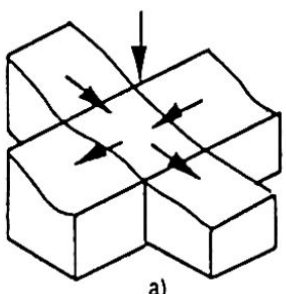

a)

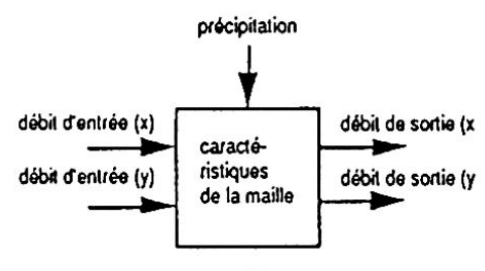

b)
Fig.1: Représentation physique des écoulements sur une maille et schématisation du traitement (d'après Abednego, 1989).

On constate aisément que des catégories de modèles de simulation et de prévision présentés, seuls ceux conceptuels régionalisés, prenant en compte la dimension spatiale des phénomènes, peuvent bénéficier de l'environnement d'un SIG. Il existe bien entendu différents niveaux d'intégration du SIG, mais de par leur nature, tous ces modèles font appel à une base de données spatialisées ou géographiques(BDG). Dans quelle mesure l'analyse spatiale à l'aide du SIG peut gérer et produire une information indispensable à ce type de modélisation? C'est ce premier niveau d'interactions qui est à étudier tout d'abord.

\section{Les descripteurs spatiaux}

Parmi les descripteurs communs pris en compte par les modèles conceptuels régionalisés, ceux considérés comme présentant des variations spatiales dans la région d'étude sont relatifs aux caractéristiques de la surface du sol. Ces dernières peuvent être décrites par la topographie et la nature de son occupation.

\subsection{La topographie}

La topographie est certainement l'information centrale pour la modélisation physique en hydrologie. Elle détermine les directions, les intensités et les modes d'écoulement de l'eau en surface ainsi que les temps d'acheminement jusqu'aux exutoires. Sa représentation numérique devra donc rendre compte le plus précisement possible de ses variations spatiales dans la zone d'étude.

De tous les descripteurs de la topographie, c'est celui de l'altitudequi est le plus fondamental. Il permet de dériver la plupart des autres indicateurs morphologiques du terrain tels que les pentes et leur orientation, les indices de forme et les bassins versants. La représentation numérique des variations de l'altitude dans la zone d'étude est appelée modèle numérique d'altitude (MNA); il est produit par des techniques d'interpolation appliquées à des données relevées généralement sur des documents tels que les cartes topographiques.

\section{La construction du modèle numérique d'altitude}

Les éléments à considérer pour la réalisation d'un modèle numérique d'altitude sont sa structure, la nature et le contenu des sources d'information disponibles, ainsi que le choix de la procédure d'interpolation adaptée aux sources et à la finalité du MNA.

\section{Source dinformation}

La carte topographique est assurément le document de description de l'altitude de plus diffusé. Il fournit des informations sur la variable d'altitude à travers les courbes de niveau et la localisation de points de valeur extrême.

\section{Structure du MNA}

La structure en un réseau maillé rectangulaire de points d'altitude offre la plus grande flexibilité d'utilisation. Elle se prête aussi bien au traitement en mode vecteur qu'à celui en mode raster. De plus, la plupart des systèmes de représentation cartographique est capable de gérer cette structure.

\section{Modélisation}

La phase de modélisation consiste à générer, à partir de l'information originale, un réseau maillé rectangulaire de points d'altitude. L'estimation de l'altitude en chacun de ces points est produite par interpolation ou extrapolation des valeurs d'altitude en des points identifiés dans le document original qui sont distribués irrégulièrement sur la surface de la zone d'étude. Le choix d'une technique d'estimation appropriée repose sur les critères suivants: 
- être adapté à la nature et à la distribution des points de mesure du document original,

- produire un MNA qui satisfait aux exigences définies pour son utilisation en hydrologie.

Pour permettre notamment la détermination de trajets d'écoulement et la délimitation des bassins versants, la procédure d'estimation doit impérativement éviter un lissage trop important et la création de minima et maxima locaux artificiels, tout en préservant ceux naturels. De telles contraintes rejettent immédiatement les méthodes d'interpolation globales ainsi que celles locales basées sur des techniques linéaires homogènes (distance pondérée, ...). Il est nécessaire de faire appel à des procédures adaptatives contenant des règles de décision basées sur le contexte spatial local.

Pour tirer profit de toute l'information fournie par la carte topographique, la procédure d'interpolation devra utiliser les valeurs d'altitude non seulement aux points relevés lors de la saisie numérique, mais tout le long des isolignes. Ce sera donc avant tout à partir d'objets linéaires constitués de lignes brisées que l'estimation sera effectuee.

La méthode retenue a été développée pour répondre à ces besoins spécifiques de l'hydrologie (ABEDNEGO 1989), elle procède de façon itérative par remplissage des points du réseau régulier à constituer. Les étapes du traitement illustrées à la Figure 2 sont les suivantes:

- Assignation des valeurs d'altitude aux points du réseau situés le long des isolignes et aux endroits d'altitude extrême relevés. Ce remplissage est réalisé à l'aide d'un algorithme de conversion vecteur-raster à partir des points d'appui saisis numériquement. Lorsque cette saisie est effectuée par un système numérique à balayage (scanner), l'assignation est opérée automatiquement. Dans cette étape, l'erreur d'estimation est ainsi inférieure à la demi-longueur d'une maille du réseau.

- Détermination des points appartenant à une zone fermée - zone limitée par une même isoligne - ou à une zone ouverte.

- Traitement des zones ouvertes: par interpolation linéaire itérative dans la direction horizontale et verticale, donnant la priorité aux points voisins d'altitude préalablement estimée. Cette distance de voisinage augmente au cours des itérations qui s'achèvent lorsque tous les points des zones ouvertes ont été traités. Les situations de «col» sont identifiées et traitées de façon spécifique afin de respecter les minima et maxima locaux naturels.

- Traitement des zones fermées: par application de la méthode B-spline. Selon l'arrangement des isolignes, ces zones constituent des cuvettes ou des collines dont la forme pourra être rendue par ce type d'interpolation à partir des points d'appui originaux et estimés lors des étapes précédentes.

- Test et correction du talweg par détermination du trajet d'écoulement dans les fonds de vallée. Dans cette étape la procédure détermine les points les plus bas de

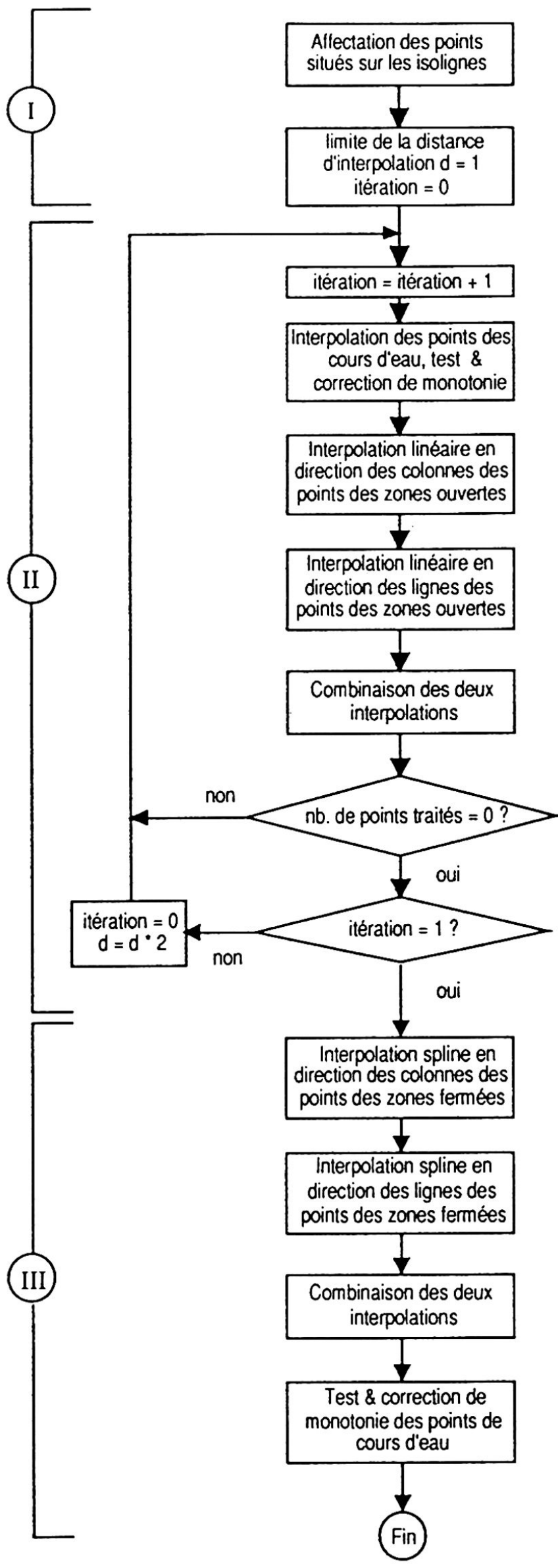

Fig. 2: Organigramme détaillant les étapes de la réalisation d'un MNA selon la méthode présentée (adapté de Abednego, 1989). 


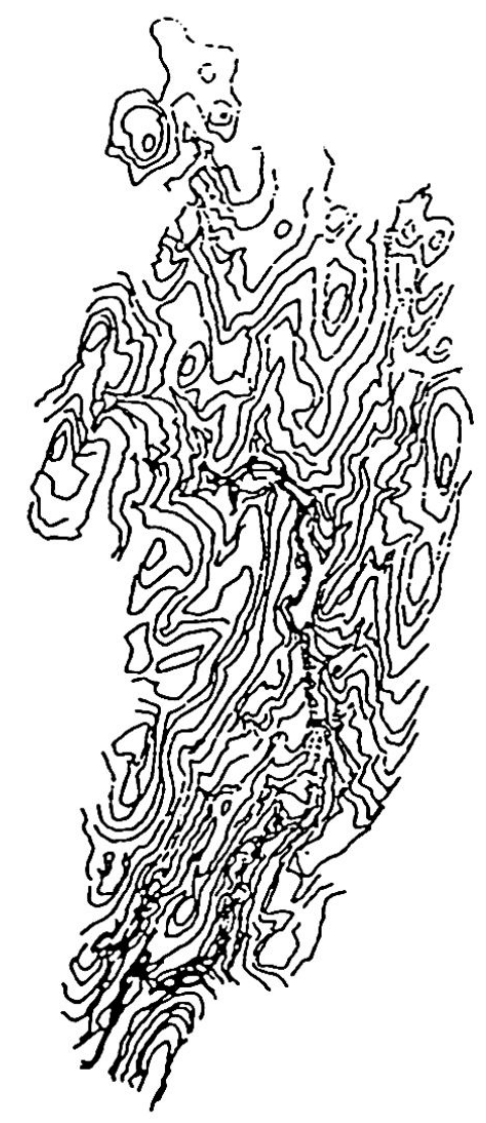

a)

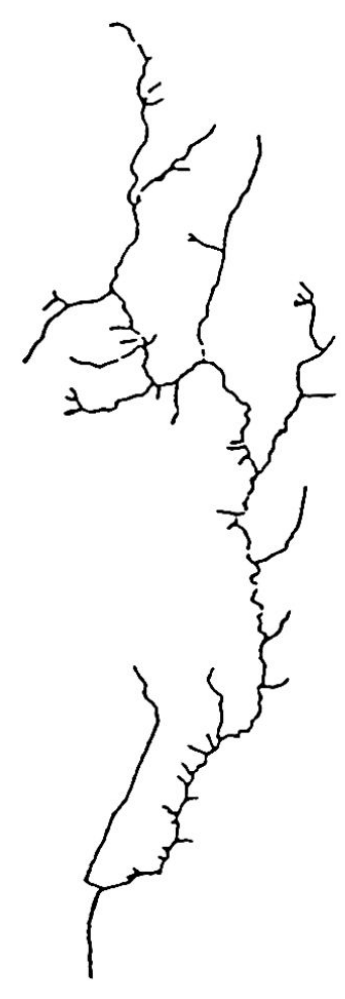

b)

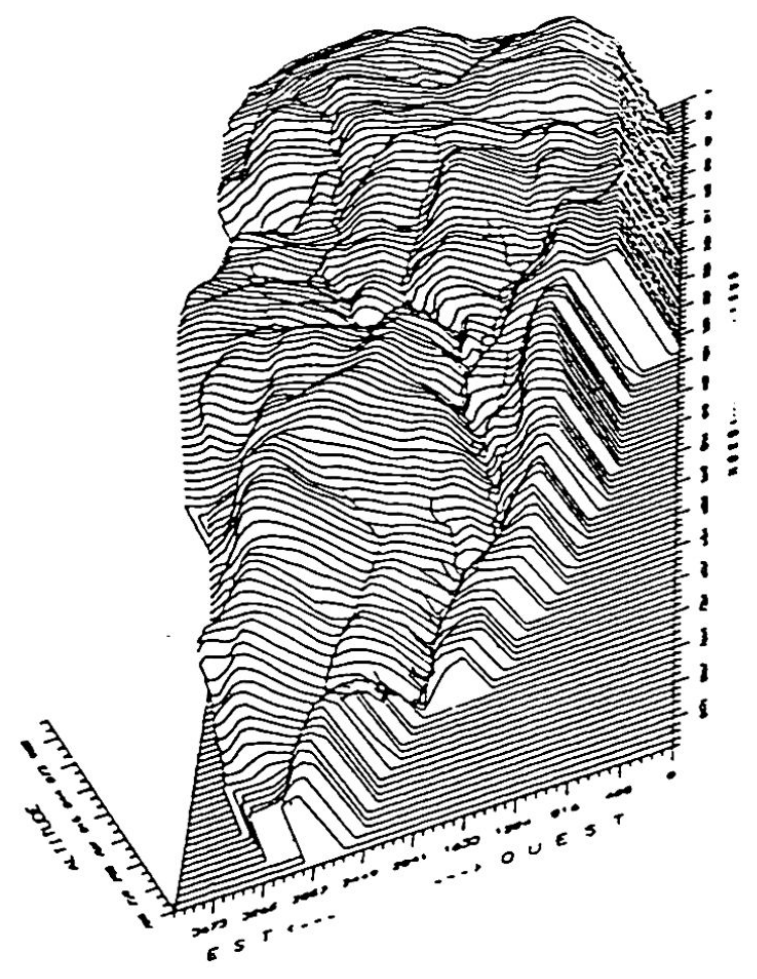

c)

Fig. 3: Application de la procédure itérative présentée à la réalisation du MNA de la Haute-Menthue (adapté de Abednego, 1989).

la topographie et corrige les maxima et minima locaux pour garantir un écoulement continu dans les talwegs. La localisation des cours d'eau figurant sur la carte topographique originale est un apport informatif significatif dans la réalisation de cette étape, il contribue à la production d'un MNA plus proche de la réalité.

La Figure 3 illustre le résultat de cette approche qui a produit un MNA de la région de la Haute-Menthue à partir d'informations numérisées de la carte topographique. Comparativement à d'autres procédures itératives telle que celle développée par l'Office fédérale de topographie à Wabern (DUFOUR, EIDENBENZ 1987) pour la réalisation du modèle numérique d'altitude DHM25, cette méthode est plus lourde en temps de traitement mais réduit de façon significative la présence des minima et maxima artificiels qui rendrait le MNA impropre à une utilisation en hydrologie.

\section{La production de descripteurs topographiques}

Parmi les nombreux descripteurs topographiques qui peuvent être dérivés d'un MNA (ANSOULT 1988), certains tels que les pentes et leur orientation, les indices de convexité du terrain, le partitionnement en bassins versants intéressent plus particulièrement l'analyse hydrologique. Une discussion détaillée de chacun d'eux sort du contexte de cet article, mais il est important de s'arrêter sur certains aspects méthodologiques. Parce qu'ils sont dérivés du MNA, leur pertinence et précision sont fonction de celles du modèle originel. De plus, les procédures d'estimation doivent être adaptées aux caractéristiques du MNA ainsi qu'à celles de leur utilisation (ABEDNEGO 1989, pp. 180-195). La plupart de ces descripteurs caractérisent non plus les points du réseau maillé, mais les surfaces comprises entre ces points. Ce passage du point à la surface s'effectue de façon implicite - mais souvent ignorée! - par les procédures opérant par filtrage spatial à partir d'un réseau maillé rectangulaire; le seul indice de cette transformation étant la production d'une grille numérique constituée d'un nombre de lignes et de colonnes amputé d'une unité (COLLET 1988).

\subsection{L'occupation du sol}

La connaissance de l'occupation du sol a un intérêt pour la modélisation hydrologique dans la mesure où elle fournit une indication quant à la rugosité de la surface du sol; 
celle-ci ayant une influence considérable sur les modalités du ruissellement et des écoulements de l'eau en surface. A ce point de vue, la télédétection satellitaire est une source d'information potentiellement riche. Il est relativement aisé de distinguer les zones de végétation des autres occupations, ainsi que d'exprimer de manière relative la densité du couvert végétal par la production d'indices de végétation. Le potentiel des images de télédétection n'est pas seulement lié à la production automatisée d'une telle information numérique et spatiale sur la rugosité, mais bien plus à la fréquence d'acquisition. Cette dernière permet de prendre en compte dans la modélisation hydrologique les modifications temporaires ou permanentes de cette rugosité de surface, donc de l'occupation du sol. D'un point de vue méthodologique, l'intégration de cette information diachronique nécessite la transformation des données de télédection multispectrale par une technique de normalisation des indices de végétation tel que le NPVI (CALOZ et al. 1986).

Cette revue des principaux descripteurs spatiaux met en évidence leur richesse informative. Un comparaison sommaire avec les catégories de modèles décrits précédemment fait apparaitre les besoins d'un couplage étroit entre les deux domaines. Si, par le passé, la liaison se réalisait par le truchement de documents cartographiques, aujourd'hui, le numérique apporte une capacité d'analyse spatiale plus grande et plus objective. Ce jugement trouve sa justification lorsque l'on examine quelques critiques émises à l'encontre des modèles globaux et maillés. On comprend alors pourquoi de nouvelles approches ont été explorées pour s'assurer de moyens de simulations à la hauteur des exigeances actuelles.

\section{Limitations des modèles globaux et mailles}

L'utilisation des modèles globaux est grandement facilitée par leur simplicité, liée au fait qu'ils ne font en général intervenir qu'un nombre très restreint de paramètres. En revanche, l'absence d'une prise en compte explicite de leur variabilité spatiale constitue un handicap dans l'analyse de l'évolution du comportement d'un bassin suite à sa modification. Ainsi, par exemple, un aménagement, selon qu'il se trouve dans la partie amont, centrale ou aval du bassin provoque des effets différents sur son comportement. Mais la différence de ces comportements ne peut pas être expliquée par des modifications des coefficients de ruissellement globaux du bassin, car ces coefficients ne reflètent pas la structure intérieure du bassin. Par exemple, une modification des types de cultures dans le bassin se répercute sur le coefficient de ruissellement global mais également sur la distribution spatiale de ce paramètre, aspect qu'il est également difficile à exprimer. Pour cette raison, les modèles de simulation hydrologiques de type global se prêtent mal à une prévision de l'impact d'une modification d'un bassin sur son comportement. Une telle prévision requiert de la part de l'utilisateur une solide expérience du modèle global utilisé de sorte qu'il soit possible de déterminer le module et les paramètres de simulation adéquats.

Au contraire de modèles globaux, les modèles maillés devraient bien convenir pour simuler les effets sur le comportement hydrologique de futurs aménagements. N'offrent-ils pas une grande souplesse pour décrire de manière précise l'occupation du sol, les types de sols avec leurs caractéristiques d'infiltration, la topographie, etc...? En pratique, ces modèles butent contre deux obstacles antagonistes qui résultent essentiellement de la taille de la maille. D'une part, pour une bonne représentation de la physique du bassin, on est conduit à choisir une maille de taille réduite et plusieurs paramètres descriptifs. L'ensemble produit un système complexe très exigent en temps de calcul et très sensible à de faibles variations de paramètres d'entrée. En effet, le grand nombre d'itérations peut produire des résultats divergeants à partir de ces faibles variations. Inconvénients auxquels il faut ajouter encore les difficultés parfois insurmontables d'acquisition des données. D'autre part, dans le cas d'une maille relativement grande (de $500 \mathrm{~m}$ à $1 \mathrm{~km}$ ou plus), les paramètres descriptifs s'éloignent d'une signification phyique précise et font perdre progressivement au modèle sa nature conceptuelle car la maille devient une unité géographique traitée au demeurant à la manière globale.

D'autres limitations des modèles de type maillé, sans aucun rapport avec la résolution spatiale, surviennent sur la représentation d'objets de type vectoriel tel qu'une canalisation. La discrétisation par mailles régulières n'est guère adaptée à ce type d'objets.

Malgré ses limites, l'approche conceptuelle conserve l'avantage d'amener l'utilisateur à une analyse spatiale poussée du bassin et de faciliter une convergence plus rapide des résultats de la simulation. Il convient de signaler encore que, pour l'hydrologue praticien, le choix d'un modèle est réalisé en référence à plusieurs critères: l'objectif de la simulation, en particulier la précision attendue, les données disponibles, les équipements informatiques et les logiciels et, souvent, sa maîtrise d'un modèle particulier. Il faut noter aussi, dans le cadre du sujet traité ici, que les données hydrologiques sont souvent très hétérogènes, certaines régions sont bien couvertes, d'autres le sont moins.

Toutes ces nouvelles exigences ont orientés les recherches vers de nouveaux concepts produits dans le sillage de l'évolution de l'informatique graphique et des logiciels d'intelligence artificielle: la modélisation orientée objet et les systèmes experts. Seul le premier cité est examiné ici.

\section{Modélisation orientée objet}

Dans son principe, la modélisation orientée objet tire les avantages de chacune des catégories présentées précédemment. L'analyse spatiale et comportementale du bassin versant conduit à le diviser en entités de type zone, 
ligne ou point dont on est capable de décrire, au moyen d'algorithmes, le fonctionnement hydrologique de manière satisfaisante. Ces entités sont appelées objets et sont décrites par des attributs et des lois de comportement. Ainsi, telle zone forestière est simulée au moyen d'un modèle global, tel élément du cours d'eau par des équations de l'hydraulique, telle zone agricole par un modèle maillé (Fig. 4).

Pour chaque objet, on choisit le modèle le plus approprié en fonction de la compréhension que l'on a de son comportement et des données disponibles. Il reste ensuite - et le problème n'est pas mineur - à agréger les effets cumulés de chaque objet. Il en résulte une grande souplesse d'utilisation ainsi qu'une optimisation simultanée des données disponibles et des performances du modèle. Cette voie n'est cependant pas simple car elle requiert de la part de l'hydrologue des compétences en matière de modèles supérieures à celles exigées par les méthodes conventionnelles. C'est pourquoi, on propose d'introduire dans la procédure d'analyse spatiale et dans celle de la sélection des modèles un système expert facilitant la tâche de l'utilisateur. (ABEDNEGO 1989)

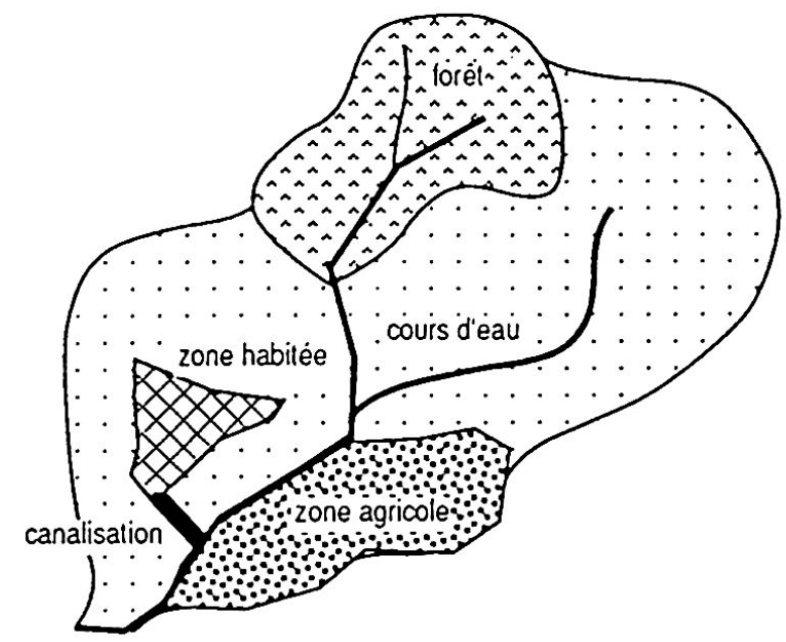

Fig. 4: Schéma d'un bassin versant découpé en objets. La schématisation du traitement est identique à celle du modèle maillé de la Figure 1.

\section{Les apports des SIG}

Selon la catégorie de modèles, les fonctions attendues du système informatique sont plus ou moins complexes. Quant aux apports d'un SIG, ils vont de l'utile à quasi l'indispensable. Examinons dans ce sens les modèles brièvement décrits.

Dans le cas d'un modèle-global «boîte noir», les informations relatives à la physique du bassin n'interviennent pas. Seules la superficie et les données hydrologiques sont retenues.

D'un point de vue informatique, l'utilisation de tels modèles peut se contenter:
- d'une base de données hydrologiques. Notons que la distribution statiale de la pluviométrie est négligée. Le modèle se satisfait d'une pluie moyenne calculée sur la base des séries chronologiques selon un temps de retour fixé au préalable

- d'un module de tracé automatique d'un bassin versant à l'aide d'un modèle numérique d'altitude décrit plus haut.

En toute évidence, la richesse et la puissance d'un SIG seraient, dans ce cas, peu valorisées. Cependant, même les utilisations toutes simples ne sont pas à negliger. Un SIG sommaire trouverait ici un rôle de nature ergonomique important comme interface graphique pour accéder facilement et de manière conviviale à la base de données et à la zone géographique de travail. On se contenterait de numériser un fond de carte et de "greffer" sur l'écran graphique des objets «sensibles» tel que le réseau hydrographique qui, activés au moyen de la souris, déclenche une procédure d'extraction des données hydrologiques ou d'autres informations nécessaires à la poursuite du travail. Ce rôle, au premier abord peu satisfaisant pour un scientifique, est tout de même important car il permettra aux utilisateurs de services publics, par exemple, de se familiariser avec les représentations géographiques informatisées et d'exploiter, en commun avec d'autres utilisateurs des données concernant le territoire.

A l'opposé, plus le modèle est de type conceptuel, plus il se nourrit de paramètres possédant, au moins de manière intrinsèque, une signification physique. La connaissance de la distribution spatiale devient alors indispensable. Un SIG apparaît alors comme un outil puissant pour accéder aux données, pour les prétraiter et extraire les paramètres requis par les modèles.

Le plus haut niveau d'interdépendance apparaît avec la modélisation orientée objet. Un SIG est d'abord utile pour les mêmes motifs qu'invoqués précédemment: repérage visuel de la zone d'analyse, interface pour accéder à la base de données établies pour le bassin versant. Il devient indispensable pour la procédure d'identification des objets hydrologiques et de détermination de leurs caractéristiques. L'analyse spatiale devient ainsi l'un des maillons les plus sensibles de l'exercice de simulation. Elle induit le choix des modèles et les opérations de regroupement des effets cumulés des éléments du bassin. Plus précisément, on peut mentionner:

- Le découpage du bassin versant en objets: sous-bassins, canalisation, réservoirs.

- L'extraction d'informations pour caractériser les objets déterminés dans l'étape précédente:

caractéristiques géographiques et géométriques des objets (emplacement, formes, dimensions)

caractéristiques physiques permettant de décrire son comportement hydrologique (section de la canalisation, coefficient de rugosité, type de couverture du sol, etc.)

- Calcul de paramètres synthétiques requis par le modèle tels que coefficient de ruissellement à partir de l'occupation du sol, calcul des limites d'un sous-bassin 
ou d'une partie de sous-bassin à l'aide d'un modèle numérique d'altitude.

- Calcul d'indicateurs généraux relatifs au bassin-versant (p.ex. pourcentage de la surface du bassin occupée par des zones imperméables) pour guider l'utilisateur dans le choix du modèle optimal.

\section{Conclusion}

Comme nous avons tenté de le montrer, certaines questions n'ont pas encore trouvé des réponses complètes et satisfaisantes. Le concept de SIG est récent et n'a probablement pas encore pu pénétrer aussi loin que l'on souhaiterait les approches méthodologiques traditionnelles de certaines disciplines. Cependant, une analyse approfondie de l'interaction SIG-Hydrologie met en évidence une indiscutable complexité du problème. En effet, l'hydrologie n'est pas une discipline des sciences exactes, elle appartient plutôt aux sciences dites naturelles, moins saisissables par des modèles mathématiques. Cet aspect est bien mis en évidence par l'inventaire, même sommaire, des modèles de simulation qui ont été conçus. Un examen attentif des modèles est également indispensable pour imaginer et comprendre l'utilisation de SIG pour leur mise en œuvre.

\section{Références bibliographiques}

ABEDNEGO, B., 1989: Apports de la télédétection à la conception de modèles de simulation en hydrologie. Thèse No. 806, EPF-L, Lausanne.

ANSOULT, M., 1988: Etude et modélisation de formes de la surface terrestre. Thèse Université Catholique de Louvain, Faculté des Sciences agronomiques, Louvain-la-Neuve, Belgique.

CALOZ, R., ABEDNEGO, B., COLLET, C., MEYLAN, P., 1986: Description of a metholdology for biomass change mapping with the use of Landsat TM data. Proceed. of IGARSS'86 Symposium, Zürich, Switzerland, pp. 1471-1476.

COLLET, C., 1988: Cartographie automatique et système d'information géographique. Notes de cours, Université de Fribourg, Suisse.

DUFOUR, D., EIDENBENZ, CH., 1987: Das digitale Höhenmodell der Landestopographie. Bundesamt für Landestopographie, Bulletin des Rechenzentrum Nr. 13, Januar 1988, Bern.

ROCHE, M.F., 1986: Dictionnaire français d'hydrologie de surface. Masson, Paris. 